\title{
Stable isotope shifted matrices enable the use of low mass ion precursor scanning for targeted metabolite identification
}

\author{
Charles B Reilly, Sri H Ramarathinam, Nicholas A Williamson, Anthony W Purcell
}

\begin{abstract}
We describe a method to identify metabolites of proteins that eliminates endogenous background by using stable isotope labeled matrices. This technique allows selective screening of the intact therapeutic molecule and all metabolites using a modified precursor ion scan that monitors low molecular weight fragment ions produced during MS/MS. This distinct set of low mass ions differs between isotopically labeled and natural isotope containing species allowing excellent discrimination between endogenous compounds and target analytes. All compounds containing amino acids that consist of naturally abundant isotopes can be selected using this scanning technique for further analysis, including metabolites of the parent molecule. The sensitivity and selectivity of this technique is discussed with specific examples of insulin metabolites identified within a complex matrix using a range of different validated low mass target ions.
\end{abstract}

\section{Background}

Mass spectrometry (MS) can identify molecules based on their distinctive mass and/or on the mass of product ions generated by fragmentation of the parent ion. It is this sensitivity and selectivity that is often exploited to distinguish a target peptide from a mixture of other closely related species [1]. The common approach for peptide identification is to use characteristic fragment ions produced during relatively mild collision activated dissociation of parent ions. The series of "sequencing" ions produced differ in mass by the residue mass of specific amino acids in addition to various neutral losses and intramolecular reactions. These ions then enable the de novo reconstruction of the primary structure of the parent peptide ion from the spectrum of fragment ions. This systematic approach relies on the prediction the fragmentation pattern of the peptide, however many variables such as collision energy and sequence composition influence fragmentation, often making it difficult to predict ion intensity relationships. This can then lead to challenges in the interpretation and prediction of MS/MS spectrum [2-5]. The uncertainty in how a

\footnotetext{
* Correspondence: apurcell@unimelb.edu.au Department of Biochemistry and Molecular Biology, The Bio21 Molecular Science and Biotechnology Institute, The University of Melbourne, Parkville, Victoria, 3010 Australia
}

particular peptide may fragment and the reliance of a classical "sequencing ions" approach to peptide identification may in some cases lead to the incomplete assignment of the spectra, where sequencing ions provide an internal sequence "tag" but fail to provide enough information for a complete peptide sequence. This is especially the case when the data from which peptide identities are made is of poor quality due to very low abundance of the parent species, or if there is only a small number of MS/MS ions present that can be used for sequencing. These sequencing difficulties are further compounded when attempting to identify peptides in very complex samples.

We propose that stable isotope labelling can improve the certainty of peptide identity. Stable isotope labelling amino acids in cell culture (SILAC), where the "heavy" isotope $\left({ }^{13} \mathrm{C},{ }^{15} \mathrm{~N}\right.$ etc.) is used for quantification of proteins by MS, is now a well-established, safe and reproducible technique [6]. The advantage of SILAC is that the only chemical difference is a single neutron that alters the atom and subsequently the amino acid mass, while not altering its biological relevance. There have also been significant advances in the complete incorporation of stable isotopes into multicellular organisms such as mice, with heavy isotope mouse feed now commercially available [6,7]. Thus the scope of experiments 
that incorporate stable isotope labelling is broad and can range from simple experiments in prokaryotes through to complex experiments in genetically modified mice. In this study we demonstrate how stable isotope labelling can be used for the discovery and identification of peptide metabolites. In contrast to similar studies where the protein or biotherapeutic is modified by incorporation of a stable isotope $[8,9]$, we propose the inverse, where the entire matrix is modified instead of the target protein. Complete incorporation of, in our case, ${ }^{15} \mathrm{~N}$ into the matrix of interest allows discrimination between matrix-derived metabolites and peptides derived from the target protein.

In addition to validating peptide identity, differential labeling can be combined with triple quadrupole technology to enable targeted data acquisition, whether in the form of multiple reaction monitoring (MRM) or during precursor ion scanning. The former technique is extremely sensitive and is currently being exploited for quantitation of known compounds; however, MRM requires knowledge of the precursor mass of the analyte which in the case of metabolites is often unknown. In contrast, precursor ion scanning can be performed without prior knowledge of the precursor mass. Combining this with the knowledge of a specific and distinguishing fragment ion can enable the use of "information dependant acquisition" (IDA) to reveal the full MS/MS of target compounds. We describe how, by using a stable isotope labeled matrix, we are able to employ the precursor ion scan to detect molecules liberated from insulin. Here the shifted matrix results in the otherwise "normal" fragment ions of the insulin becoming the unique and distinguishing features that can be harnessed to trigger targeted MS/MS analysis.

The target ions used in this case are low mass ions that are below $200 \mathrm{~m} / z$; these ions include, but are not limited to, the immonium and related ions and can be indicators for the presence of corresponding unlabeled amino acid residues (Table 1). Currently low mass ions are under-exploited in peptide identification; however, recent studies such as Hohmann et al [10] suggest that the immonium ion can be used as a quantitative indicator. The presence and relative intensity of the immonium ion reflect not only the presence of a particular amino acid residue but can also describe where within the peptide the residue is located. In our study we are particularly interested in the immonium ion due to its relationship to peptide amino acid composition. The immonium ion can form through various contextdependant mechanisms but consists of the general structure $\mathrm{RCH}=\mathrm{NH}_{2}{ }^{+}$with $\mathrm{R}$ being the side chain of the amino acid residue. This results in a defined set of immonium ions (and related ions that have undergone an additional fragmentation in the form of a neutral loss) that represent all of the 20 common amino acids. The particular feature of these ions that has been exploited in this study is that the immonium ions always contain at least one nitrogen as a remnant from the amide bond found in all peptides. This has meant that with the complete incorporation of ${ }^{15} \mathrm{~N}$ in a metabolic matrix, the immonium ions derived from endogenous peptides found within the matrix will not be observable at the normal $m / z$.

During this study we tested the ability to selectively screen out specific peptides from a ${ }^{15} \mathrm{~N}$ complex mixture using immonium ions corresponding to amino acid residues found within a target peptide. Figure 1 shows the well characterized Glu Fibrinopeptide EGVNDNEEGFFSAR (GluFib) being selectively screened from a complex mixture. Here 1 pmol of GluFib is added to a complex matrix consisting of $40 \mu \mathrm{g}{ }^{15} \mathrm{~N}$ compounds taken from the acid soluble fraction of $E$. coli which had been cultured exlusively in ${ }^{15} \mathrm{~N}$ media. A multiple precursor ion scan that simultaneously monitors for more than one target ion (in this case two) is employed to target the MS analysis towards GluFib. The target ions used are 72 and 120 and represent the amino acid residues of valine and phenylalanine respectively. During this analysis the entire matrix was ignored and signal detection from the precursor scan was only obtained from unlabeled GluFib, thus triggering a targeted MS/MS While at 1 pmol concentration it is possible to identify GluFib within this matrix using a conventional LC-MS/MS approach, the differential isotope labeling enables high confidence during characterization with conventional sequencing. In addition, only MS/MS spectra belonging to GluFib is provided, reducing the data load for search algorithms.

The real advantage of this strategy is in situations where target molecules coelute with many compounds of higher intensity. We therefore directly compared the targeted precursor scan data acquisition to untargeted LC-MS/MS where GluFib was titrated into a ${ }^{15} \mathrm{~N}$ complex mixture $\left(40 \mu \mathrm{g}{ }^{15} \mathrm{~N}\right.$ as above). In Figure 2 (i, A-C)) we show the total ion chromatogram (TIC) of all three product ion spectra acquired as a result of IDA during a single LC-MS/MS when $50 \mathrm{fmol}$ of GluFib is added to the complex mixture. At this concentration GluFib is completely ignored and not selected for product ion acquisition during untargeted LC-MS/MS. In contrast, when the same sample was run with identical LC and ion source conditions, but with a precursor ion scan monitoring for an immonium ion for valine $(72 \mathrm{~m} / z)$, GluFib was efficiently detected. The TIC for the precursor scan in Figure 2 (ii, D-F) shows a strong and distinct signal when GluFib is present despite the enhanced mass spectrum (EMS) that was obtained during IDA showing low abundance of the target ion (785.7 amu, Figure 2F), relative to all other ions present. During this 
Table 1 List of low mass target ions in native and stable isotope labelled peptides

\begin{tabular}{|c|c|c|c|}
\hline Residue & Native Immonium ion & Related native ions & ${ }^{15} \mathrm{~N}$ Immonium ion \\
\hline Alanine, A & 44 & & 45 \\
\hline Arginine, $R$ & 129 & $59,70,73,87,100,112$ & 133 \\
\hline Asparagine, $\mathrm{N}$ & 87 & 70 & 89 \\
\hline Apartic acid, D & 88 & 70 & 89 \\
\hline Cysteine, C & 76 & & 77 \\
\hline Glutamic acid, E & 102 & & 103 \\
\hline Glutamine, Q & 101 & $56,84,129$ & 103 \\
\hline Glycine, G & 30 & & 31 \\
\hline Histidine, $\mathrm{H}$ & 110 & $82,121,123,138,166$ & 113 \\
\hline |soleucine, I & 86 & 44,72 & 87 \\
\hline Phenylalanine, F & 120 & 91 & 121 \\
\hline Proline, $\mathrm{P}$ & 70 & & 71 \\
\hline Leucine, L & 86 & 44,72 & 87 \\
\hline Lysine, K & 101 & $70,84,112,129$ & 103 \\
\hline Methionine, M & 104 & 61,70 & 105 \\
\hline Serine, $\mathrm{S}$ & 60 & & 61 \\
\hline Threonine, $T$ & 74 & & 75 \\
\hline Tryptophan, W & 159 & $77,117,130,132,170,171$ & 161 \\
\hline Tyrosine, Y & 136 & 91,107 & 137 \\
\hline Valine, V & 72 & $41,55,69$ & 73 \\
\hline \multicolumn{2}{|c|}{ Insulin tryptic peptides } & \multicolumn{2}{|c|}{ Target ions used for precursor scanning } \\
\hline \multicolumn{2}{|c|}{ FVNQHLCGSHLVEALYLVCGER } & \multicolumn{2}{|c|}{$120(\mathrm{~F}), 70(\mathrm{P} / \mathrm{R} / \mathrm{N}), 86(\mathrm{~L} / \mathrm{l}), 72(\mathrm{~V})$} \\
\hline \multicolumn{2}{|c|}{ GFFYTPKT } & \multicolumn{2}{|c|}{$120(\mathrm{~F}), 70(\mathrm{P} / \mathrm{R} / \mathrm{N})$} \\
\hline \multicolumn{2}{|c|}{ GIVDQCCTSICSLYQLENYCN } & \multicolumn{2}{|c|}{$72(\mathrm{~V}, 86(\mathrm{~L} / \mathrm{l}), 70(\mathrm{P} / \mathrm{R} / \mathrm{N})$} \\
\hline
\end{tabular}

(Top) List of immonium and related ions for the 20 naturally abundant amino acids and the expected isotopic shifts of immonium ions when $15 \mathrm{~N}$ labeled. (Bottom) Tryptic Insulin digest peptides and the potential target ions to be used for information dependant MS/MS acquisition (IDA) targets during a precursor ion scan.

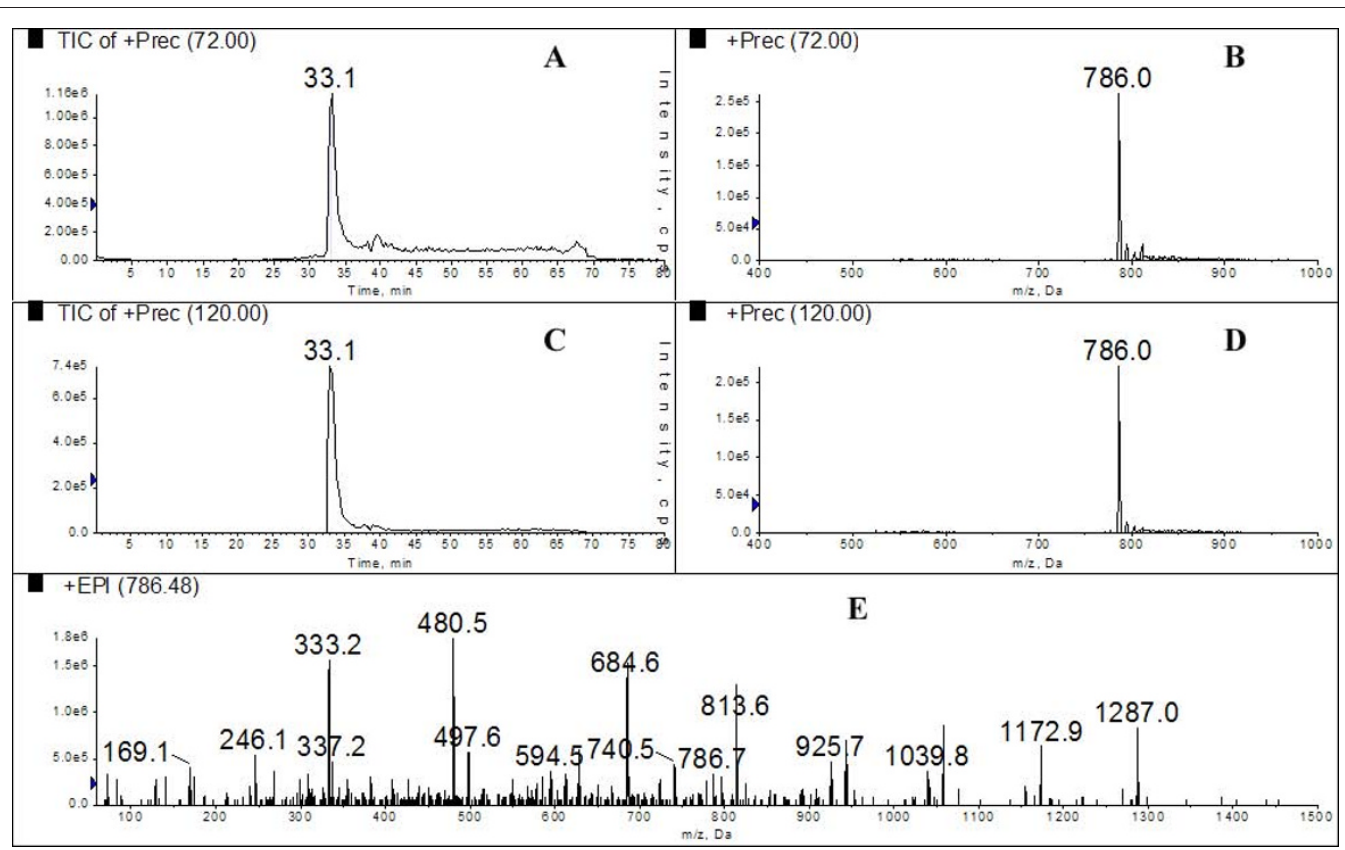

Figure 1 Multiple precursor ion scanning for GluFib from within a complex mixture. 1 pmol of GluFib was added to $40 \mu \mathrm{g}{ }^{15} \mathrm{~N}$ matrix consisting of the acid soluble fraction of $E$. coli. A double precursor ion scan was performed using the immonium ion targets of (A) 72 (valine) and (C) 120 (phenylalanine). Figure 1, shows the precursor ion scans both detect the $786 \mathrm{~m} / \mathrm{z}$ ion (B and D respectively) and subsequently trigger IDA of a product ion spectra (E) that produces sequencing ions for the GluFib peptide EGVNDNEEGFFSAR. 


\section{(i) LC-MS/MS}

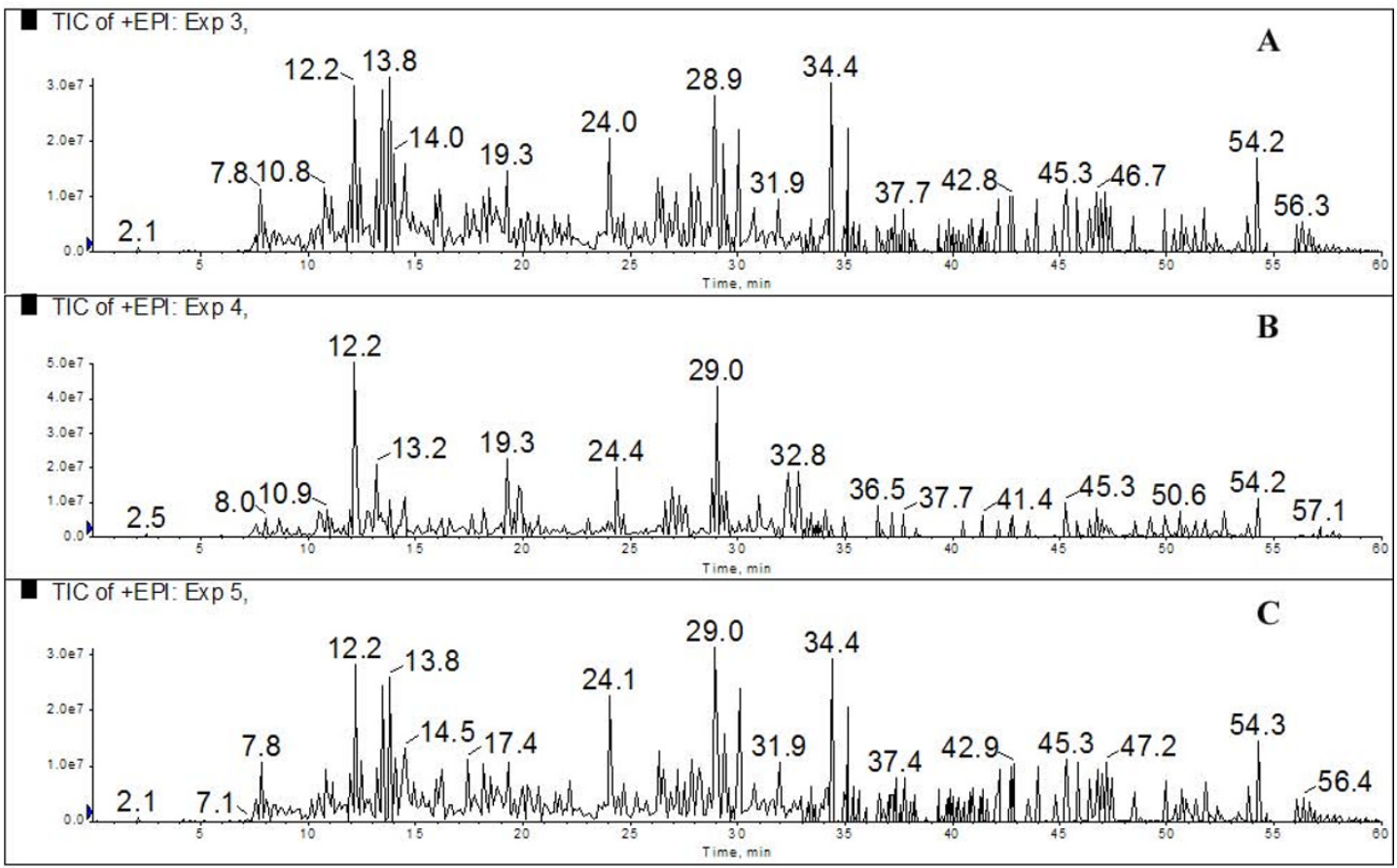

(ii)

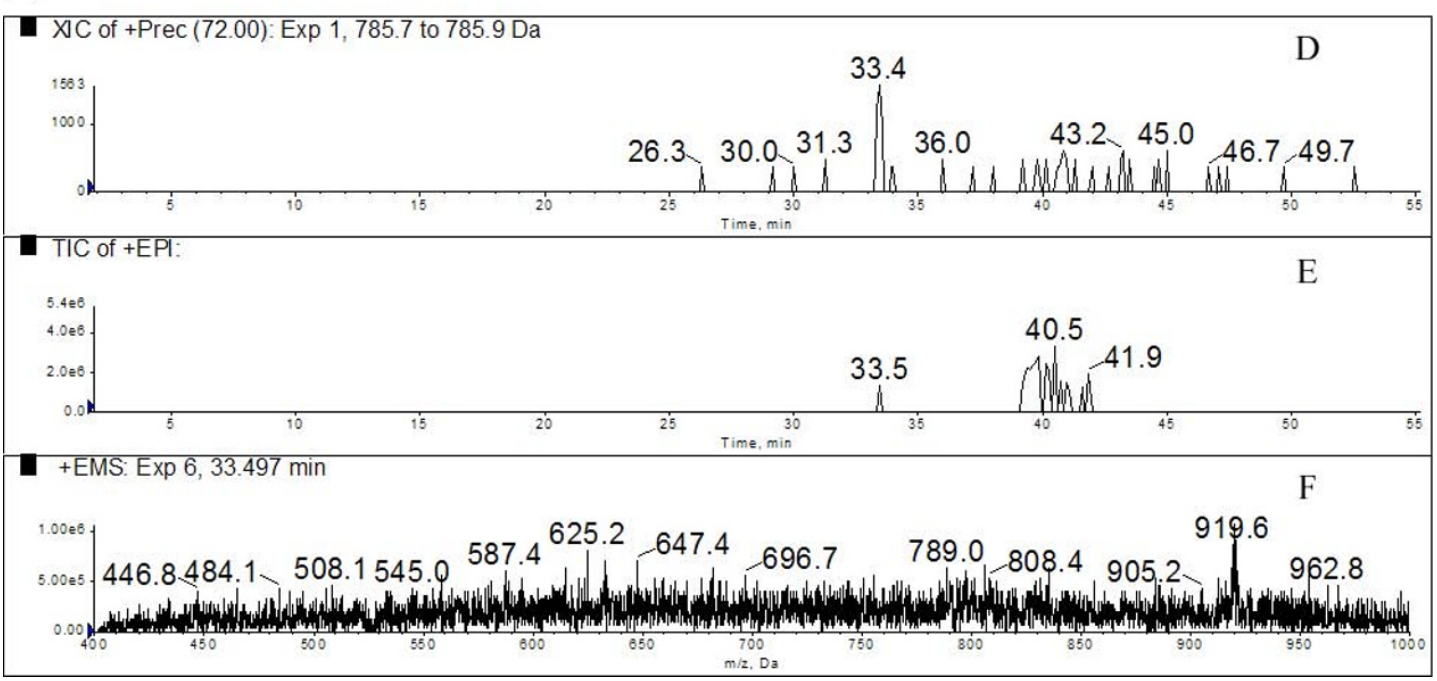

Figure 2 Precursor ion scanning for GluFib from a ${ }^{15} \mathrm{~N}$ complex mixture below the detection level of a traditional LC-MS/MS approach $100 \mathrm{fmol}$ of GluFib was spiked into $80 \mu \mathrm{g}$ (total protein) of a ${ }^{15} \mathrm{~N}$ matrix consisting of the acid soluble fraction of E. coli and split into two. One half was analysed as a regular LC-MS/MS (i) while the other as a Precursor scan for $\mathrm{m} / \mathrm{z} 72$ valine (ii). At this concentration of GluFib the regular LC-MS/MS did not select the target peptide for MS/MS due to the complexity of the sample. In contrast the precursor scan identified GluFib and automatically selected it for MS/MS data acquisition. The LC-MS/MS experiment consisted of an IDA that selects the three most intense ions from each mass spectrum for product ion acquisition. (i) Shows the TIC of all three product ion spectra acquired during the single LC-MS/MS experiment, none of which selected the precursor mass of $786 \mathrm{~m} / z$ (GluFib) for product ion acquisition due to the complexity of the sample. In contrast the precursor scan (ii) shows the product ion spectra TIC (E) with a peak at 33.5 mins, the precursor ion triggering IDA at this time point had an $\mathrm{m} / \mathrm{z} 786$, corresponding to GluFib and appropriately product ion spectra was acquired for confirmation. (F) The total mass spectrum corresponding to the time point when GluFib was detected and triggered product ion data acquisition, highlighting the low abundance of the GluFib $\mathrm{m} / \mathrm{z} 786$ ion relative to the complex background. (D) Extracted ion chromatogram (XIC) for the GluFib precursor mass from the precursor scan for $\mathrm{m} / \mathrm{z} 72$ showing the most intense signal during the elution of GluFib. (The precursor ion scan chromatograms are cropped to highlight the time when GluFib is eluted.) 
analysis no signal was obtained and thus no product ion spectra acquired until the time point $33.4 \mathrm{~min}$, which is consistent with the highest concentration of GluFib during the chromatographic elution when an extracted ion chromatogram is performed on the precursor ion scan (Figure 2D).

Furthermore, the selection of the internal residues valine and phenylalanine illustrates the strength of the approach through its ability to employ a variety of low mass ions that can come from residues found throughout the target peptide.

Lastly this targeted approach to metabolite identification is especially relevant to the monitoring and discovery of biotherapeutic metabolites in complex matrices where targets are of unknown precursor mass. Here we took peptides derived from a tryptic digest of injectable insulin and added them to the same complex matrix mentioned above (40 $\mu \mathrm{g}{ }^{15} \mathrm{~N}$ as above). We observed specificity and selectivity for peptides liberated from insulin by virtue of proteolysis by bacterial peptidases contained within the $E$. Coli lysate. The same sample was also run as a standard LC-MS/MS experiment and both results submitted to a ProteinPilot version 4 search algorithm. While the LC-MS/MS came back with no positive identifications for insulin, the precursor scan identified the predicted tryptic peptides as well as additional unexpected insulin peptides. These included peptides with modified cysteine residues and truncated peptides that were the result of residual protease activity within the ${ }^{15} \mathrm{~N}$ E. coli matrix.

The experimental cycle loop of the 4000 QTrap instrument used consisted of precursor ion scans that targeted up to four specific immonium ions, with the detection of any one of these ions prompting IDA.

Base Peak Chrom. of +EMS

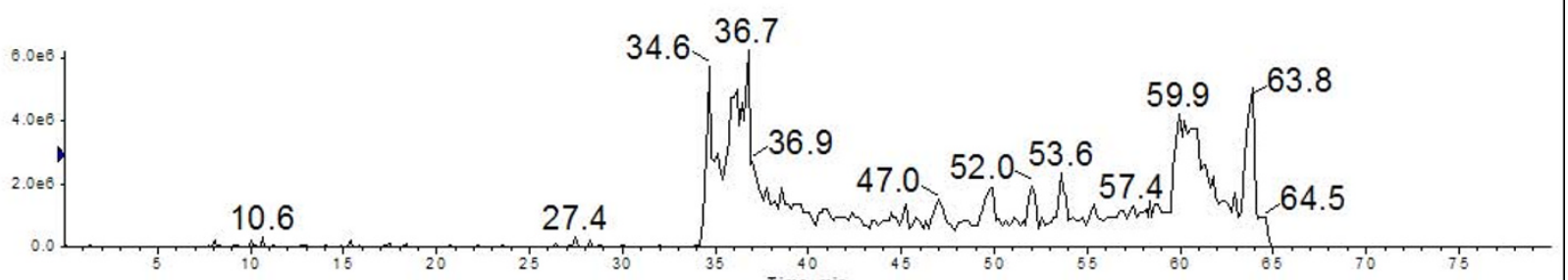

\begin{tabular}{l|l} 
TIC of +Prec (86.10) & Time. $\min$ \\
$+\operatorname{Prec}(86.10)$ & 50.606
\end{tabular}

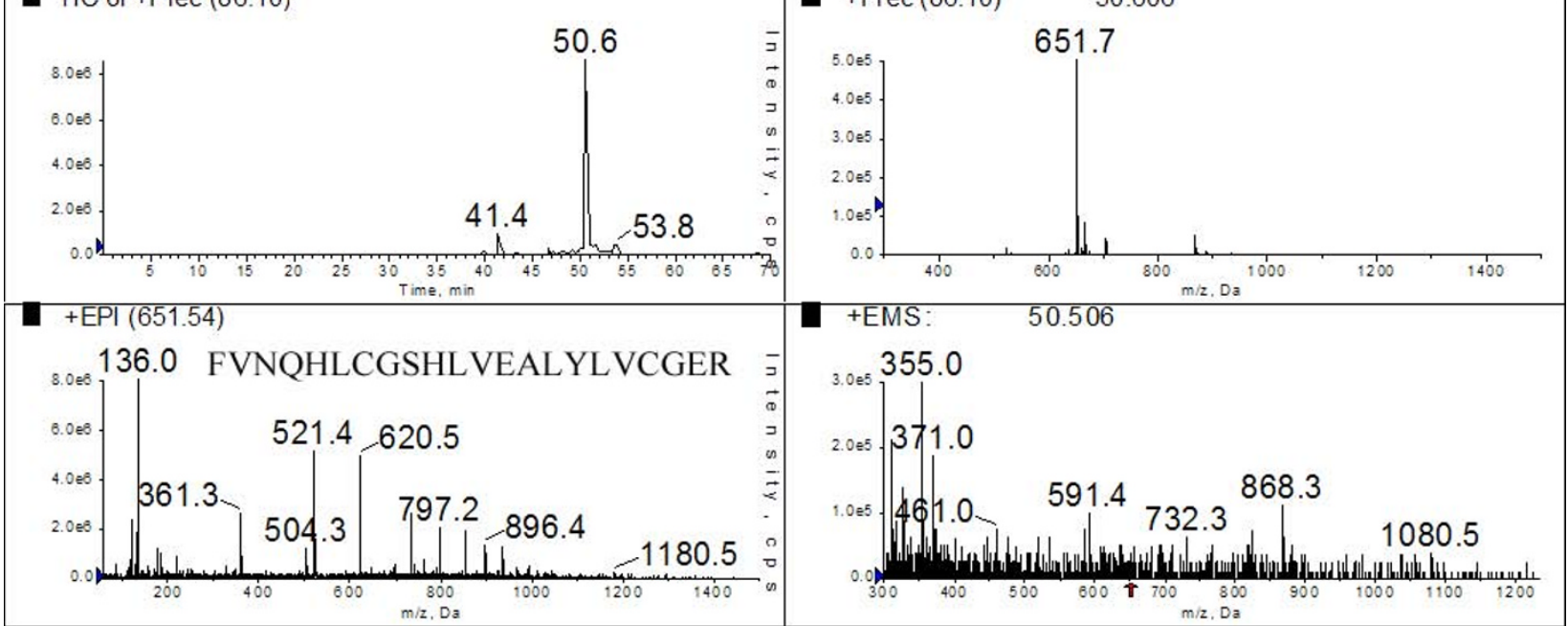

Figure 3 Insulin peptides were combined with a ${ }^{15} \mathrm{~N}$ complex matrix. 2 pmol of an insulin tryptic digest was added to $40 \mu \mathrm{g}$ (total protein) of a ${ }^{15} \mathrm{~N}$ matrix, consisting of the acid soluble fraction of $E$. coli. The same sample was run as a regular LC-MSMS experiment and as precursor ion scanning experiments. Target ions during the precursor scans consisted of immonium and related ions corresponding to residues $L$, I, $Y, V, N$, $D$ and F. (A) The base peak chromatogram during the LC-MS/MS procedure. (B) The total ion chromatogram (TIC) for the precursor ion scan with target ion $86 \mathrm{~m} / \mathrm{z}$ which corresponds to lle and Leu. (C) The precursor scan at time point 50.6 min (which corresponds to the major peak in the TIC) showing the presence of an intense peak for $651.5 \mathrm{~m} / \mathrm{z}$ which corresponds to the insulin peptide FVNQHLCGSHLVEALYLVCGER. (D) The mass spectrum at the 50.6 min time point showing that the $651.5 \mathrm{~m} / \mathrm{z}$ ion is of low abundance relative to the complex spectral background. (E) The Product ion spectrum for the ion at $\mathrm{m} / \mathrm{z} 651.5$ which was acquired due to detection of a target ion during the precursor scan at time point $50.6 \mathrm{~min}$. The sequencing ions lead to a positive identification of the peptide FVNQHLCGSHLVEALYLVCGER. Both the LC-MS/MS and precursor ion scan data sets were submitted to the same ProteinPilot version 4 search engine. Only the Precursor ion scan identified peptides liberated from insulin. 
This in turn resulted in acquisition of an enhanced product ion spectrum and an EMS. Figure 3 shows a total ion chromatogram (TIC) for the precursor scan for $\mathrm{m} / z$ 86 (immonium ion corresponding to leucine/isoleucine) and a major peak at time point $50.6 \mathrm{~min}$. At this time point the major precursor mass that was giving a signal for the presence of an $86 \mathrm{~m} / z$ was 651.7 and accordingly an IDA-triggered EPI was generated. The MS/MS data in this spectrum corresponds to that of the insulinderived tryptic-like peptide FVNQHLCGSHLVEALYLVCGER (Table 2). In addition to the EPI we included an EMS to be triggered during the precursor scan in order to provide context. Figure 3 (bottom right panel) shows the mass spectrum at the same time point for when the $651 \mathrm{~m} / z$ peptide was identified (50.6 min). Here we see that the peptide would not have been observed during a conventional MS/MS acquisition due to the large number of ions with much greater ion intensity than the insulin peptide signal. These data shows that despite the target peptide being in low abundance relative to the complex spectral background it still gives the highest intensity signal during the precursor scan, resulting in "targeted data acquisition" for a peptide that was derived from insulin regardless of matrix complexity. When the data acquired was subjected to analysis by ProteinPilot version 4 the only successful protein ID was insulin.

Table 2 Sequencing ions for the insulin peptide FVNQHLCGSHLVEALYLVCGER

\begin{tabular}{|c|c|}
\hline b ions & $y$ ions \\
\hline 148.1 & 2601.3 \\
\hline 247.1 & 2454.2 \\
\hline 361.2 & 2355.1 \\
\hline 489.2 & 2241.1 \\
\hline 626.3 & 2113.1 \\
\hline 739.4 & 1976.0 \\
\hline 899.4 & 1862.8 \\
\hline 956.4 & 1702.9 \\
\hline 1043.5 & 1645.8 \\
\hline 1180.5 & 1558.8 \\
\hline 1293.6 & 1421.7 \\
\hline 1392.7 & 1308.7 \\
\hline 1521.7 & 1209.6 \\
\hline 1592.8 & 1080.6 \\
\hline 1705.8 & 1009.5 \\
\hline 1868.9 & 896.4 \\
\hline 1981.1 & 733.4 \\
\hline 2081.1 & 620.3 \\
\hline 2241.1 & 521.2 \\
\hline 2298.1 & 361.2 \\
\hline 2427.2 & 304.2 \\
\hline 2583.3 & 175.1 \\
\hline
\end{tabular}

\section{Summary}

We have shown that by using stable isotope labeled matrices combined with precursor ion scanning and monitoring low mass ions, we are able to selectively screen for peptides liberated from proteins of interest. The precursor scanning technique assumes no prior knowledge or prediction of how the products within that matrix will form and is able to specifically screen peptides that may even contain unanticipated post translational modifications. The selectivity employed in triple quadrupole technology allows for targeted fragmentation data acquisition for the peptides/metabolites of interest at low abundance relative to a complex endogenous background. Furthermore, the data acquired is readily searchable using current informatics algorithms without a requirement to analyze MS/MS data containing ${ }^{15} \mathrm{~N}$ or alternative stable isotopes. This technique will find many applications in monitoring changes to biomolecules - especially in relation to biotherapeutics within complex sample mixtures. Moreover, with the advent of isotopically labeled animal feed one can envision the use of this technique for absorption, distribution, metabolism, and excretion (ADME) type studies of peptide and protein drugs in experimental animals.

\section{Authors' contributions}

CBR performed the majority of the experiments, conceived the study, contributed to experimental design and drafted the manuscript. SHR provided technical assistance and mass spectrometry support. NAW provided expert mass spectrometry guidance. AWP conceived the study, participated in it design and coordination and co-wrote the manuscript. All authors read the final manuscript.

\section{Competing interests}

The authors declare that they have no competing interests.

Received: 8 June 2010 Accepted: 17 January 2011

Published: 17 January 2011

\section{References}

1. Purcell AW, Gorman JJ: Immunoproteomics: Mass Spectrometry-based Methods to Study the Targets of the Immune Response. Mol Cell Proteomics 2004, 3:193-208.

2. Bleiholder C, Osburn S, Williams TD, Suhai S, Van Stipdonk M, Harrison AG, Paizs B: Sequence-scrambling fragmentation pathways of protonated peptides. J Am Chem Soc 2008, 130:17774-17789.

3. Harrison AG: Peptide sequence scrambling through cyclization of $b(5)$ ions. J Am Soc Mass Spectrom 2008, 19:1776-1780.

4. Paizs B, Suhai S: Fragmentation pathways of protonated peptides. Mass Spectrom Rev 2005, 24:508-548.

5. Paizs B, Suhai S, Harrison AG: Experimental and theoretical investigation of the main fragmentation pathways of protonated $\mathrm{H}$ Gly-Gly-Sar-OH and H-Gly-Sar-Sar-OH. J Am Soc Mass Spectrom 2003, 14:1454-1469.

6. Ong SE, Blagoev B, Kratchmarova I, Kristensen DB, Steen H, Pandey A, Mann M: Stable isotope labeling by amino acids in cell culture, SILAC, as a simple and accurate approach to expression proteomics. Mol Cell Proteomics 2002, 1:376-386.

7. Kruger M, Moser M, Ussar S, Thievessen I, Luber CA, Forner F, Schmidt S, Zanivan S, Fassler R, Mann M: SILAC mouse for quantitative proteomics uncovers kindlin-3 as an essential factor for red blood cell function. Cell 2008, 134:353-364. 
8. Colzani M, Bienvenut W, Faes E, Quadroni M: Precursor ion scans for the targeted detection of stable-isotope-labeled peptides. Rapid

Communications in Mass Spectrometry 2009, 23:3570-3578.

9. Williamson NA, Reilly C, Tan C-T, Ramarathinam S-H, Jones A, Hunter CL, Rooney FR, Purcell AW: A novel strategy for the targeted analysis of protein and peptide metabolites. PROTEOMICS 2010, EPub DEC 152010.

10. Hohmann LJ, Eng JK, Gemmill A, Klimek J, Vitek O, Reid GE, Martin DB:

Quantification of the compositional information provided by immonium ions on a quadrupole-time-of-flight mass spectrometer. Anal Chem 2008, 80:5596-5606.

doi:10.1186/1477-5956-9-2

Cite this article as: Reilly et al: Stable isotope shifted matrices enable the use of low mass ion precursor scanning for targeted metabolite identification. Proteome Science 2011 9:2.

Submit your next manuscript to BioMed Central and take full advantage of:

- Convenient online submission

- Thorough peer review

- No space constraints or color figure charges

- Immediate publication on acceptance

- Inclusion in PubMed, CAS, Scopus and Google Scholar

- Research which is freely available for redistribution

Submit your manuscript at www.biomedcentral.com/submit
() Biomed Central 